\title{
Traumatismo encefalocraneano en pediatría
}

\author{
ADRIANA WEGNER A. ${ }^{1}$, PAMELA CÉSPEDES F. ${ }^{2}$ \\ 1. Médico Intensivista pediátrico, Jefe de la Unidad de Paciente Crítico Pediátrico, Complejo Asistencial Dr. Sótero del Río. \\ 2. Médico Pediatra, Residente de la Unidad de Paciente Crítico Pediátrico, Complejo Asistencial Dr. Sótero del Río.
}

\begin{abstract}
Traumatic brain injury in pediatrics

Traumatic Brain Injury (TBI) is an important Public Health issue in Chile and the world. It represents a frequent cause of consultation, constituting a significant cause of morbidity and mortality in the population under 45 years of age. Accidents are the main reason for TBI among the pediatric population, but child abuse is an important cause in children below 2 y.o. A proper evaluation is essential to develop timely and efficient treatment that avoids or decreases brain damage and eventual complications. For this purpose, it is essential that brain physiology and physiopathological changes triggered by TBI are clear and well known. Current concepts are presented in this paper, emphasizing brain hemodynamics, metabolism, and brain self-regulation.

(Key words: Traumatic brain injury, children, brain physiology, brain circulation, brain self-regulation, brain metabolism, brain edema).
\end{abstract}

Rev Chil Pediatr 2011; 82 (3): 175-190

\section{RESUMEN}

El traumatismo encefalocraneano (TEC) es un importante problema de salud pública tanto en Chile como en el mundo. Representa un motivo de consulta frecuente constituyendo una de las mayores causas de morbimortalidad en la población menor de 45 años. Los accidentes son la principal causa de TEC en la población pediátrica, pero el maltrato infantil es una causa etiológica importante a considerar en los menores de 2 años. Realizar una correcta evaluación al paciente con TEC es fundamental para instaurar un tratamiento oportuno y eficiente con el fin de evitar y/o disminuir el daño cerebral y así prevenir eventuales complicaciones. Para ello es imprescindible el conocimiento de la fisiología cerebral y los cambios fisiopatológicos que se desencadenan posterior al TEC, conceptos que son revisados en este artículo con énfasis en la hemodinamia cerebral, metabolismo y autorregulación cerebral.

(Palabras clave: Traumatismo encefalocraneano, niños, fisiología cerebral, circulación cerebral, autorregulación cerebral, metabolismo cerebral, edema cerebral).

Rev Chil Pediatr 2011; 82 (3): 175-190

Trabajo recibido el 18 de marzo de 2011, devuelto para corregir el 21 de abril de 2011, segunda versión el 02 de mayo de 2011, aceptado para publicación el 09 de mayo de 2011.

Correspondencia a:

Adriana Wegner A.

E-mail: awegnera@gmail.com 


\section{Introducción}

El TEC se define como un intercambio brusco de energía mecánica causado por una fuerza externa que tiene como resultado una alteración a nivel anatómico $\mathrm{y} / \mathrm{o}$ funcional (motora, sensorial y/o cognitiva) del encéfalo y sus envolturas, en forma precoz o tardía, permanente o transitoria. El TEC se diferencia de la contusión craneal en que en esta no se produce alteración del contenido intracraneal. La alteración del contenido encefálico se refiere al compromiso de conciencia, la amnesia postraumática $\mathrm{y} / \mathrm{o}$ a un síndrome vertiginoso o mareos persistentes. Se considera también como un signo de disfunción del contenido craneal la aparición de una cefalea holocránea persistente y progresiva que puede o no acompañarse de vómitos. Particularmente en lactantes se debe considerar un equivalente de inconsciencia la presencia de palidez asociada a inmovilidad ${ }^{1}$.

E1 TEC puede clasificarse de diferentes maneras: según tipo de lesión encefálica (difusa o focal, figura 1); según indemnidad meníngea (TEC abierto y cerrado); según tipo de frac-

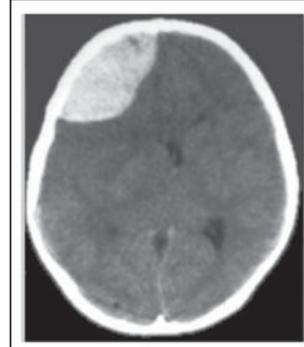

1a

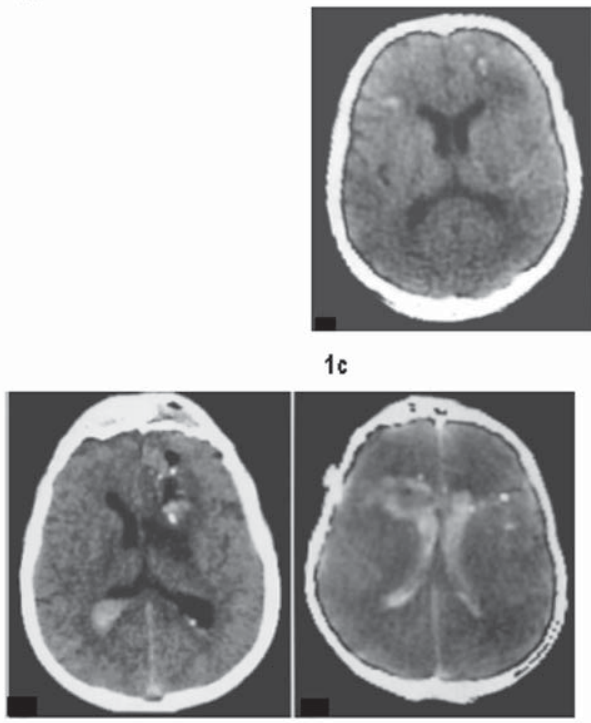

1d

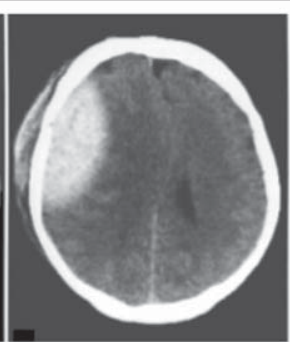

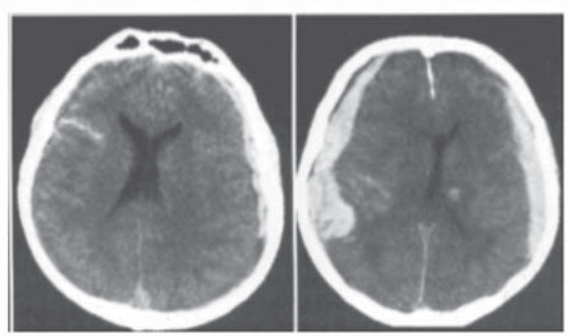

$1 \mathrm{~b}$
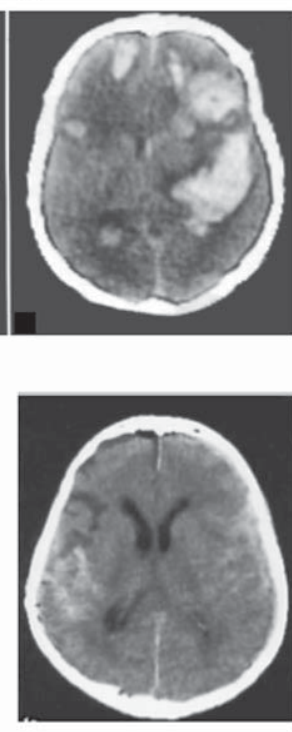

1e

Figura 1. Lesiones intracraneanas focales. 1a. Hematoma epidural o extradural: colección de sangre entre la duramadre y el cráneo, que puede ser de origen venoso o arterial, generalmente no sobrepasa líneas de las suturas a menos que coexista con fractura. Al TAC aparece como una imagen localizada, lenticular, de alta densidad, con evidente efecto de masa. 1b. Hematoma Subdural: colección de sangre localizada sobre la superficie de la corteza cerebral, bajo la duramadre, que es de origen venos. Al TAC se observa una imagen crescéntica, hiperdensa, localizada a lo largo de las convexidades cerebrales. 1c. Hematoma intracerebral: coágulo sólido denso dentro del parénquima cerebral. 1d. Hemorragia intraventricular: presencia de sangre al interior de los ventrículos. Implica mecanismo de alta energía. 1e. Hemorragia subaracnoidea: presencia de sangre entre piamadre y aracnoides. 
Tabla 1. Escala de Glasgow

a. Escala de Glasgow

\begin{tabular}{|llllll|}
\hline Apertura ocular & & Respuesta motora & \multicolumn{3}{c|}{ Respuesta verbal } \\
\hline Espontánea & 4 & Espontánea, normal & 6 & Orientada & 5 \\
\hline A la voz & 3 & Localiza al tacto & 5 & Confusa & 4 \\
\hline Al dolor & 2 & Localiza al dolor & 4 & Palabras inapropiadas & 3 \\
\hline Ninguna & 1 & Decorticación & 3 & Sonidos incomprensibles & 2 \\
& & Descerebración & 2 & Ninguna & 1 \\
\hline
\end{tabular}

b. Escala de Glasgow modificada para lactantes

\begin{tabular}{|llllll|}
\hline Apertura ocular & & Respuesta motora & \multicolumn{3}{c|}{ Respuesta verbal } \\
\hline Espontánea & 4 & Espontánea, normal & 6 & Arrullos, balbuceos & 5 \\
\hline A la voz & 3 & Localiza al tacto & 5 & Irritable & 4 \\
Al dolor & 2 & Localiza al dolor & 4 & Llora al dolor & 3 \\
Ninguna & 1 & Decorticación & 3 & Quejido al dolor & 2 \\
& & Descerebración & 2 & Ninguna & 1 \\
& Ninguna & 1 & & \\
\hline
\end{tabular}

tura (TEC con fractura de base de cráneo o con fractura bóveda craneal); según grado de compromiso neurológico aplicando la Escala de Glasgow (tabla 1). Esta última representa la clasificación de mayor relevancia clínica pues permite dirigir el manejo general ${ }^{2,3}$. Así se reconoce: TEC Leve, puntaje Escala Glasgow (GCS) 13-15; TEC Moderado, puntaje escala Glasgow 12-9 y TEC Grave, puntaje escala Glasgow $\leq 8$ o caída del GCS en 2 o más puntos en 1 hora.

\section{Epidemiología}

El TEC constituye un motivo de consulta frecuente en los Servicios de Urgencia y es la causa más frecuente de mortalidad en la edad escolar. De acuerdo a la Asociación de Sociedades Científicas-Médicas de Chile ASOCIMED, por cada fallecido existirían 45 hospitalizaciones y 1300 consultas, con una tasa de incidencia de 200 por cada 100000 niños al año, de los cuales un $81 \%$ es catalogado como TEC leve y un $14 \%$ como moderado o severo. Entre un 3\% a un 53\% de los pacientes con TEC leve presentarán lesiones intracranea- nas en la Tomografía Axial Computadorizada (TAC), lo que incluye al grupo de pacientes con GCS de 15 quienes tendrían un riesgo de lesión intracraneal de 2,5 a 7\%. Sin embargo, sólo un 0,3 a $4 \%$ de todos estos pacientes requerirá de una intervención quirúrgica inicial. Por otro lado, la incidencia de deterioro tardío en pacientes con lesiones cefálicas se estima entre 1 a 4\%, aunque esta situación es extremadamente rara en pacientes con TEC leve y TAC y examen neurológico inicial normal, describiéndose en este grupo un riesgo menor al $0,5 \%$ de, incluso, complicaciones no significativas. Una excepción a esto lo constituyen los menores de 1 año, quienes deben considerarse siempre una población de alto riesgo. Adicionalmente, la letalidad del TEC se relaciona con el Glasgow inicial, reportándose un $2 \%$ a $3 \%$ en los casos moderados y alrededor de $30 \%$ o más en el caso de los graves. Sobre el $97 \%$ de los pacientes con GCS de 3 mueren o quedan en estado vegetativo persistente ${ }^{4-6}$.

Respecto a las causas de TEC, si bien en adultos la mayoría corresponde a accidentes automovilísticos y asaltos, en los niños son secundarias a caídas domésticas, accidentes de tránsito y recreacionales (accidentes en bici- 
cleta y deportivos). Una causa importante de TEC a considerar en los niños menores de 2 años, es el maltrato infantil o trauma no accidental cuya incidencia se estima en 15 por 1000 niños por año, reportándose en Estados Unidos una tasa de fallecimientos por esta causa de 1000 muertes por año ${ }^{4-8}$.

Existen particularidades en el TEC pediátrico respecto al de adultos, pues en la edad pediátrica el desarrollo del cráneo y su contenido definen los tipos de lesiones más frecuentes ${ }^{6-7}$ :

- En los lactantes el mayor tamaño de la cabeza y la debilidad de los músculos del cuello determinan que este grupo tenga mayor riesgo de lesiones provocadas por mecanismos de rotación y aceleración-desaceleración. La capacidad elástica del cráneo y las suturas abiertas predisponen a que la hemorragia cerebral progrese sin signos iniciales evidentes provocando shock hemorrágico. Además, el alto contenido de agua y viscosidad del cerebro los hace más propensos a desarrollar un daño axonal.

- Es más frecuente el TEC cerrado y las principales lesiones asociadas son: (a) hemorrágicas focales y lesiones no hemorrágicas que comprometen la sustancia gris cortical; (b) daño axonal difuso y (c) daño secundario causado por edema y colecciones ocupantes de espacio.

- Las lesiones quirúrgicas por efecto de masa (ej., hematomas subdurales y epidurales) se producen con menos frecuencia $y$, cuando se presentan, se asocian a una menor mortalidad.

- El TEC grave tendría una menor frecuencia con una mortalidad también menor 6 .

- El deterioro rápido después de un período lúcido inicial (síndrome Talk and Die) es también menos frecuente respecto a adultos (3\% frente a $10 \%)$.

- Existe un mayor grado de recuperabilidad en el tiempo en los niños producto de la mayor plasticidad neuronal de sus cerebros en desarrollo.

- Las convulsiones postraumáticas precoces ocurren con mucha más frecuencia en niños y, a su vez, son más frecuentes en los niños más pequeños que en los niños mayores.
- El síndrome de migraña postraumática se observa esencialmente en la edad pediátri$\mathrm{ca}^{6}$.

- El síndrome post-conmoción es característico en la edad pediátrica y puede causar confusión con una lesión de masa en expansión.

\section{Fisiología y alteraciones en el TEC}

Clásicamente el daño que se produce en el TEC se divide en injuria primaria e injuria secundaria.

La Injuria Primaria consiste en la disrupción directa del parénquima cerebral inmediatamente producido el trauma, generando zonas de penumbra o en riesgo de convertirse en zonas dañadas. La Injuria primaria es imposible de revertir pues las neuronas no se regeneran una vez dañadas o muertas, por lo que sólo la Prevención Primaria es efectiva en este contexto $^{6-8}$.

La Injuria Secundaria, se refiere al daño que se produce posterior a la injuria primaria por desarrollo de eventos intracerebrales y/o eventos extracerebrales como la hipoxia y/o la hipotensión, estos últimos factores de mal pronóstico demostrado para un mal resultado neurológico de no ser revertidos rápida y oportunamente. Los eventos producidos durante la injuria secundaria son potencialmente manejables, por lo que las acciones terapéuticas deben dirigirse a evitar o minimizar las cascadas metabólicas gatilladas por la injuria inicial con el fin de disminuir los riesgos de una mala evolución neurológica y/o muerte ${ }^{6-8}$.

El daño neuronal inicial desencadena una serie de alteraciones anatómicas, celulares y moleculares que perpetúan la injuria. Entre estas se cuentan la disrupción de la microvasculatura (estenosis, pérdida de vasculatura), ruptura de la barrera hematoencefálica (BHE) por inflamación de los podocitos astrocitarios (también puede romperse en la lesión primaria), proliferación de astrocitos (astrogliosis) con la consiguiente captación reversa de glutamato que determina depolarización neuronal mediante mecanismos de exitotoxicidad y alteración del influjo de calcio intracelular, el cual es el puntapié inicial en una serie de cascadas 
moleculares que resultan en disfunción y/o muerte neuronal y desconexión neuronal tardía. En las neuronas el influjo de calcio a través de la activación de los receptores NMDA y AMPA por glutamato provocará exitotoxicidad, stress oxidativo (generación de radicales libres), disfunción mitocondrial con falla en los mecanismos de generación de energía (ATP) y modificaciones de los receptores post sinápticos. La acumulación de calcio dentro de los axones iniciará una serie de cascadas de degradación de proteínas que determinará la desconexión axonal. Las células inflamatorias también mediarán el daño en la injuria secundaria a través de aumentar citoquinas proinflamatorias (leucotrienos, interleuquinas) que contribuyen a la activación de cascadas de muerte celular/apoptosis o modificaciones de receptores postsinápticos. Todos estos procesos determinarán regeneración cicatricial y edema cerebral, con la consiguiente isquemia e hipertensión intracraneana (HTEC) secundarias $\mathrm{y}$, finalmente, necrosis y apoptosis celular (figura 2) 9-11.

Para evitar y/o disminuir el daño secundario es necesario conocer y entender la fisiología cerebral normal y las alteraciones que se producen posteriores a un TEC, de manera tal de instaurar las medidas terapéuticas racional y oportunamente.
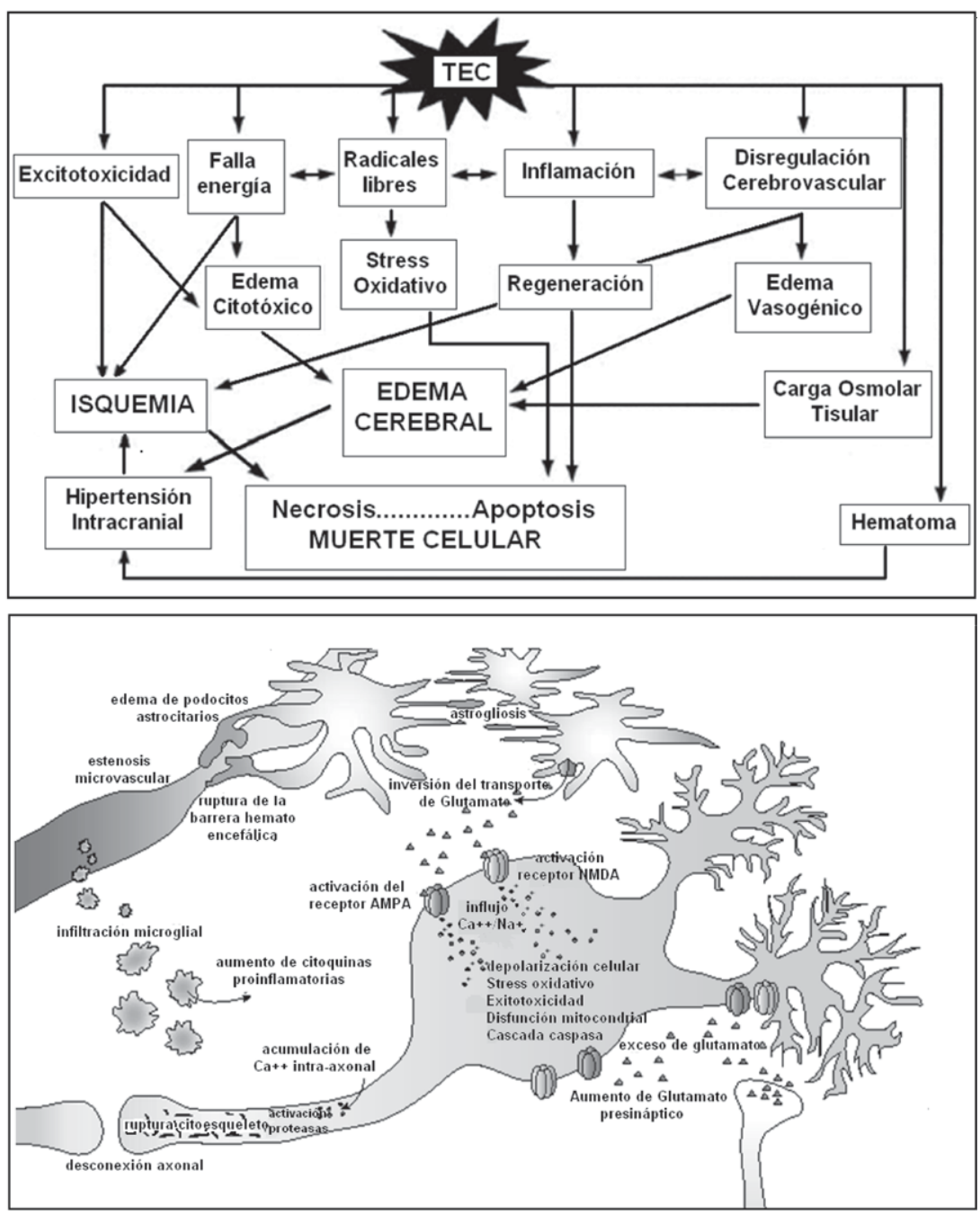

Figura 2. Mecanismos fisiopatológicos en el TEC. Adaptado de: Kochanek PN, Clark RSB, Ruppel RA, et al: Biochemical, cellular and molecular mechanisms in the evolution of secondary damage after severe traumatic brain injury in infants and children: Lessons learned from the bedside. Pediatr Crit Care Med 2000; 1: 4-19; Park E, Bell JD, Baker AJ. Traumatic brain injury: Can the consequences be stopped? CMAJ 2008; 178 (9): 1163. 


\section{Flujo sanguíneo cerebral, presión de perfusión cerebral, presión intracraneana}

El Flujo sanguíneo cerebral (FSC) es controlado por la tasa metabólica de oxígeno cerebral $\left(\mathrm{CMRO}_{2}\right)$, la autorregulación de la resistencia vascular cerebral (RVC) y por la presión de perfusión cerebral (PPC) que es la diferencia entre la presión arterial media (PAM) y la Presión Intracraneana (PPC $=$ PAM -PIC). Así, el FSC $=\mathrm{PPC} / \mathrm{RVC}$ o FSC $=(\mathrm{PAM}-\mathrm{PIC})$ $\mathrm{RVC}^{12}$.

La PPC, esto es, la presión con la que se perfunde el tejido nervioso, requiere de valores mínimos para asegurar un adecuado funcionamiento cerebral. Se ha determinado que una PPC $<40 \mathrm{mmHg}$ se asocia consistentemente con una mortalidad elevada, independiente de la edad ${ }^{13}$. No es claro si este valor representa un umbral mínimo o si la PPC óptima puede estar por encima de esto en niños (por ejemplo, 50-65 $\mathrm{mmHg}$ ), aunque es probable que se relacione con la edad, pues se conoce que una PPC baja podría ser bien tolerada en cerebros inmaduros (recién nacidos). En adultos se ha definido que una PPC $>60 \mathrm{mmHg}$ puede ser adecuada para mantener un buen funcionamiento cerebral debiendo evitarse valores $<50$ $\mathrm{mmHg}^{14}$.

La PIC varía con la edad, posición corporal y condición clínica. En adultos en posición supina la PIC varía entre 7 y $15 \mathrm{~mm} \mathrm{Hg}$ y en la posición vertical es negativa con un promedio de $-10 \mathrm{mmHg}$ sin exceder los $-15 \mathrm{mmHg}$. En niños la PIC normal oscilaría entre 3 y $7 \mathrm{~mm}$ $\mathrm{Hg}$ y en recién nacidos y lactantes entre 1,5 y $6 \mathrm{mmHg}^{14}$. Se habla de HTEC cuando los valores de PIC superan los valores normales para la edad, aunque la recomendación general es iniciar tratamiento con PIC $\geq 20 \mathrm{mmHg}$, si bien existen autores que recomiendan utilizar umbrales menores en niños: $15 \mathrm{mmHg}$ en lactantes, $18 \mathrm{mmHg}$ en niños menores de 8 años y de $20 \mathrm{mmHg}$ en niños mayores ${ }^{15-16}$.

La PIC depende del volumen intracraneal (VI) y de la interacción entre sus componentes: cerebro, líquido cefalorraquídeo (LCR) y sangre. El cerebro constituye el $80 \%$ de la bóveda craneana y se considera un compartimiento inextensible pues está compuesto en un
$75-80 \%$ por agua la que mayoritariamente se encuentra en el intracelular (sustancia blanca acomoda $\geq 10 \%$ del agua). El LCR corresponde al 10\% del VI, es modificable por terapias; se produce en los plexos coroideos a una tasa de $0,35 \mathrm{ml} / \mathrm{min}$ o $500 \mathrm{ml} /$ día, se reabsorbe en las vellosidades aracnoideas y su cantidad promedio es de 90-150 ml. La sangre corresponde al $10 \%$ del VI y está compuesto por el Volumen sanguíneo cerebral (VSC) y por el FSC. El VSC corresponde a la sangre presente constantemente en el cerebro y contribuye en forma directa a la PIC y al VI. El FSC es la sangre que fluye transitoriamente en el cerebro y que afecta a la PIC indirectamente mediante la autorregulación cerebral y que es modificable en forma terapéutica ${ }^{7,12,17}$.

La relación PIC-VI tiene un comportamiento sigmoideo, lo que implica que por sobre los valores considerados como normales leves aumentos del VI provocará grandes cambios de PIC, esto será más marcado dependiendo de la cronicidad del aumento de VI (figura 3$)^{15}$.

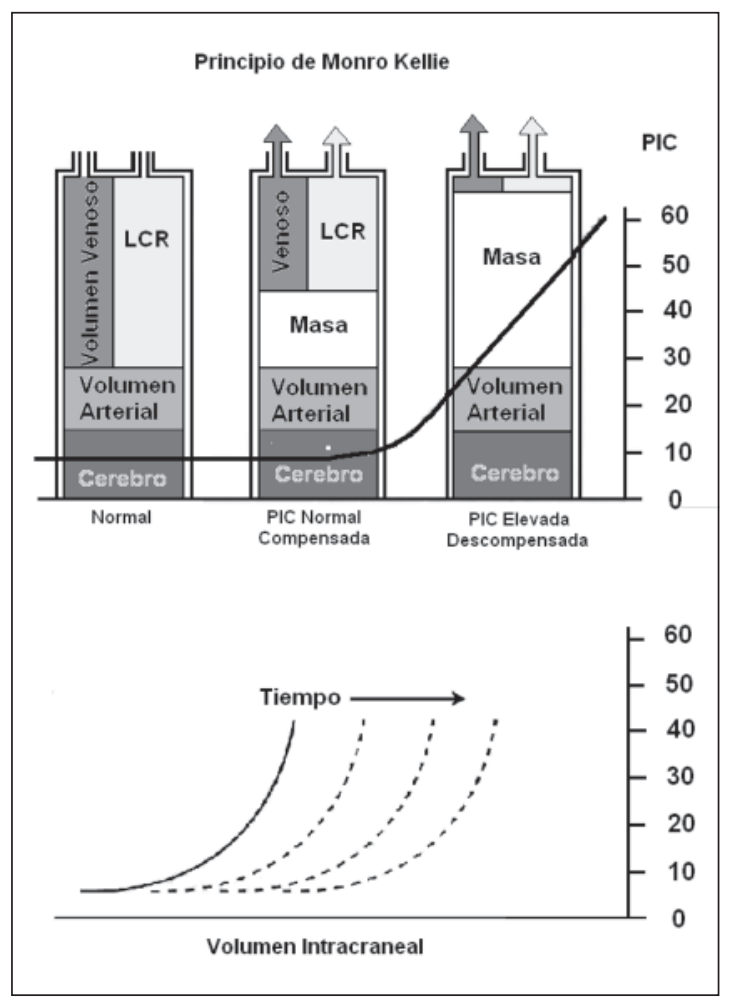

Figura 3. Relación PIC Volumen Intracraneal. 


\section{Metabolismo cerebral}

El FSC representa el 15\%-25 del gasto cardíaco, tres cuartas partes del cual se dirige a la sustancia gris y el resto a la sustancia blanca $(80 \mathrm{ml} / 100 \mathrm{~g}$ tejido cerebral/min versus 20 $\mathrm{ml} / 100 \mathrm{~g}$ tejido cerebral/min $)^{12,18-20}$. El FSC se ha estimado mediante la Ultrasonografía Doppler Transcraneal midiendo la velocidad del flujo sanguíneo (VFS) en las arterias cerebrales. Con este método se ha determinado que los neonatos tendrían una baja VFS $(24 \mathrm{~cm} / \mathrm{s})$ la que aumentaría con la edad alcanzando un peak entre los 6 y 9 años $(97 \mathrm{~cm} / \mathrm{s})$, comenzando a disminuir después de los 10 años hasta equipararse con los valores del adulto (50 $\mathrm{cm} / \mathrm{s}$ ) y que la VFS sería mayor en el grupo de mujeres respecto al de los hombres en casi todos los grupos etarios (tabla 2). De esta mane$\mathrm{ra}$, se ha estimado que los adultos tendrían un FSC promedio de aproximadamente $50 \mathrm{ml} / 100$ $\mathrm{g} / \mathrm{min}$, valor que es más elevado en niños y adolescentes y que es menor en neonatos, esto es, alrededor de $20 \mathrm{ml} / 100 \mathrm{~g}$ tejido cerebral/ min cifra extremadamente baja respecto a la proporción cerebro- peso corporal comparado con adultos (tabla 3 ).

Respecto a los valores críticos de FSC, se describe que el daño neuronal es irreversible y tiempo-dependiente con un FSC $<10-15$ $\mathrm{ml} / 100 \mathrm{~g} / \mathrm{min}$ pudiendo existir reversibilidad con un FSC > 15 a $20 \mathrm{ml} / 100 \mathrm{~g} / \mathrm{min}$, datos que han sido extrapolados de estudios en adultos.

Por otro lado, la $\mathrm{CMRO}_{2}$ o nivel de consumo de oxígeno del cerebro, es en promedio de $3,2 \mathrm{ml} \mathrm{O}_{2} / 100 \mathrm{~g} / \mathrm{min}$ en adultos, del cual 2/3 corresponde al consumo de la sustancia gris y $1 / 3$ al de la sustancia blanca. Al igual que el FSC varía con la edad, siendo mayor en niños y adolescentes y bastante menor en neonatos $(2,3 \mathrm{ml} / 100 \mathrm{~g} / \mathrm{min}$ en lactantes, $5,2 \mathrm{ml} / 100 \mathrm{~g} /$ min entre los 3 y 11 años y 4,2 ml/100 g/min en jóvenes). Algo semejante es lo que ocurre con la tasa metabólica de glucosa cerebral: es más baja en neonatos (13-25 $\mu \mathrm{mol} / 100 \mathrm{~g} / \mathrm{min})$, aumenta progresivamente con la edad alcan-

Tabla 2. Flujo sanguíneo cerebral estimado por la velocidad de flujo sanguíneo mediante ecotomografía Doppler transcraneal

\begin{tabular}{|ccccccc|}
\hline Edad & Niños & Niñas & Ambos & Niños & Niñas & Ambos \\
\hline $0-10 d$ & --- & --- & $24 \pm 7$ & --- & --- & --- \\
$11-90 d$ & --- & --- & $42 \pm 10$ & --- & --- & -- \\
\hline $3-11,9 m$ & --- & --- & $74 \pm 14$ & --- & -- & $51 \pm 6$ \\
\hline $1-2,9 a$ & --- & --- & $85 \pm 10$ & --- & $70 \pm 10$ & $58 \pm 6$ \\
\hline $3-5,9 a$ & $92 \pm 13$ & $99 \pm 11$ & $94 \pm 10$ & $61 \pm 9$ & --- & $58 \pm 9$ \\
\hline $6-9,9 a$ & --- & --- & $97 \pm 9$ & --- & $59 \pm 11$ & $46 \pm 8$ \\
\hline $10-16,9 a$ & $75 \pm 16$ & $89 \pm 16$ & $81 \pm 11$ & $51 \pm 12$ & 59 \\
\hline
\end{tabular}

$V_{a c m}$ : velocidad de flujo arteria cerebral media; $V_{a b}$ : velocidad de flujo arteria basilar. Adaptado de: Udomphorn $Y$, Armstead W, Vavilala M. Cerebral Blood Flow and Autoregulation After Pediatric Traumatic Brain Injury. Pediatr Neurol 2008; 38: $225-34$.

Tabla 3. Flujo sanguíneo cerebral y $\mathrm{CMRO}_{2}$ en adultos y neonatos

\begin{tabular}{|llll|}
\hline Edad & Peso cerebral & FSC & $\mathbf{C M R O}_{\mathbf{2}}$ \\
Neonato & $\begin{array}{l}\text { aprox } 350-400 \mathrm{~g} \\
(10 \% \text { peso corporal) }\end{array}$ & $\begin{array}{l}15-20 \mathrm{ml} / 100 \mathrm{~g} / \mathrm{min} \\
(3,4-4,8 \mathrm{tt} / \mathrm{h})\end{array}$ & $1,1-2,1 \mathrm{ml} / 100 \mathrm{~g} / \mathrm{min}$ \\
& $1200-1400 \mathrm{~g}$ & $40-50 \mathrm{ml} / 100 \mathrm{~g} / \mathrm{min}$ & $(248-472 \mathrm{ml} \mathrm{O} / \mathrm{h})$ \\
Adulto & $(2 \%$ peso corporal) & $(31,2-39,0 \mathrm{lt} / \mathrm{h})$ & $3,2 \mathrm{ml} / 100 \mathrm{~g} / \mathrm{min}$ \\
& & $(2500 \mathrm{ml} \mathrm{O} / \mathrm{h})$ \\
\hline
\end{tabular}


zando un peak a los 3-4 años (49-65 $\mu \mathrm{mol} / 100$ $\mathrm{g} / \mathrm{min}$ ) y se mantiene alta hasta alrededor de los 9 años, para posteriormente equipararse con el valor de adultos $(19-33 \mu \mathrm{mol} / 100 \mathrm{~g} /$ min) (figura 4).

Las variaciones etarias observadas en el FSC, $\mathrm{CMRO}_{2}$ y el CMR glucosa responderían a la menor resistencia cerebrovascular existente en los niños y adolescentes respecto a adultos y neonatos $(0,8 \mathrm{~mm} \mathrm{Hg} / 100 \mathrm{~g} / \mathrm{min}$ entre los 3 y 11 años versus $1,4 \mathrm{mmHg} / 100 \mathrm{~g} / \mathrm{min}$ en jóvenes).

Respecto al $\mathrm{CMRO}_{2}$, el $40 \%$ responde al gasto energético basal, básicamente a la mantención del potencial de membrana, el cual es termosensible y no modificable por drogas y el $60 \%$ restante responde al gasto energético funcional, el cual no es termosensible y es modificable por drogas. Adicionalmente, el $90 \%$ del $\mathrm{CMRO}_{2}$ se concentra en el tejido neuronal y el $10 \%$ en el tejido de sostén o glia que corresponde a más del $50 \%$ del volumen encefálico.

El $\mathrm{CMRO}_{2}$ depende del Coeficiente de extracción cerebral de $\mathrm{O}_{2}\left(\mathrm{CERO}_{2}\right)$ y de la entrega cerebral de $\mathrm{O}_{2}\left(\mathrm{CDO}_{2}\right)$, lo que se relaciona de la siguiente manera: $\mathrm{CMRO}_{2}=\mathrm{CERO}_{2} \mathrm{x}$ $\mathrm{CDO}_{2}$. La $\mathrm{CERO}_{2}$ se define por la diferencia entre la Saturación arterial de $\mathrm{O}_{2}\left(\right.$ Sat art $\left.\mathrm{O}_{2}\right)$ y la Saturación venosa en el Bulbo Yugular (Sat $\mathrm{BYO}_{2}$ ), que no es más que la sangre venosa de retorno cerebral y que varía entre $55-70 \%$. Así, la $\mathrm{CERO}_{2}$ puede estimarse de la siguiente manera $\mathrm{CERO}_{2}=\left(\right.$ Sat art $\mathrm{O}_{2}-$ Sat $\left.\mathrm{BYO}_{2}\right) /$ Sat art $\mathrm{O}_{2}$, todos valores medibles en forma clínica y que permitirían estimar el grado de consumo cerebral de $\mathrm{O}_{2}$ del cerebro y con ello su estado metabólico.

Por otro lado, la $\mathrm{CDO}_{2}$ está determinada por el FSC y por el Contenido Arterial de $\mathrm{O}_{2}$ $\left(\mathrm{CaO}_{2}\right)$, relacionándose de la siguiente manera: $\mathrm{CDO}_{2}=\mathrm{FSC} \times \mathrm{CaO}_{2}$. El $\mathrm{CaO}_{2}$ : depende de la Sat art $\mathrm{O}_{2}$, de la saturación de la hemoglobina y su concentración y de la presión parcial de $\mathrm{O}_{2}$ arterial: $\mathrm{CaO}_{2}=$ Sat $\mathrm{O}_{2} \times[1,34 \times \mathrm{Hb}]+$ $\left[\mathrm{PaO}_{2} \times 0,003\right]$. Así, cualquier disminución en la cantidad de $\mathrm{O}_{2}$ (ej. anemia, hipoxemia, etc.) y/o en el FSC determinará una $\mathrm{CDO}_{2}$ inadecuada.

Entonces, el $\mathrm{CMRO}_{2}=\left(\mathrm{FSC} \times \mathrm{CaO}_{2}\right) \times($ Sat art $\mathrm{O}_{2}$-Sat $\left.\mathrm{BYO}_{2}\right) /$ Sat art $\mathrm{O}_{2}$, factores que deben ser vigilados y monitorizados para asegurar el adecuado suplemento de oxígeno para el buen funcionamiento cerebral.

\section{Control de la circulación cerebral}

La circulación cerebral (FSC) está estrechamente regulada por mecanismos homeostáticos, tales como la presión arterial de $\mathrm{CO}_{2}$ $\left(\mathrm{PaCO}_{2}\right)$, la presión arterial de $\mathrm{O}_{2}\left(\mathrm{PaO}_{2}\right)$, el metabolismo y la viscosidad sanguínea.

$\mathrm{La} \mathrm{PaCO}_{2}$ es un potente vasodilatador cerebral y en adultos por cada aumento de $1 \mathrm{mmHg}$

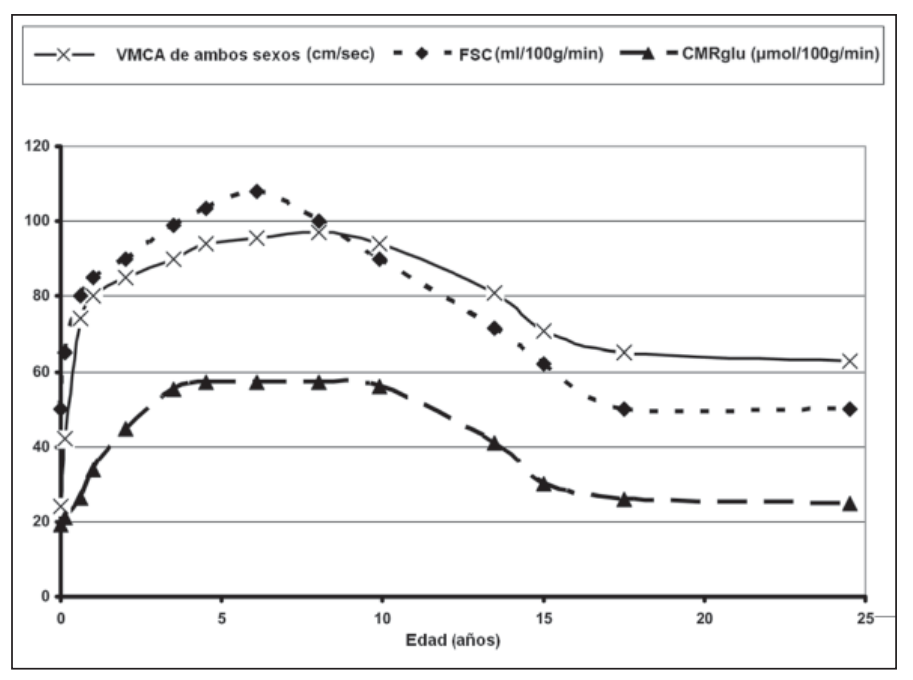

182
Figura 4. Evolución de $\mathrm{FSC}, \mathrm{CMRO}_{2}$ y $\mathrm{CMR}$ glucosa con la edad. VMCA: Velocidad de Arteria Cerebral Media; CBF: Flujo sanguíneo cerebral; CMRglu: tasa metabólica de glucosa. Adaptado de: Udomphorn, Y; Armstead, W; Vavilala, M. Cerebral Blood Flow and Autoregulation After Pediatric Traumatic Brain Injury. Pediatr Neurol 20085; 38: 225-34. 
en la $\mathrm{PaCO}_{2}$ en el rango de $25-75 \mathrm{mmHg}$ el FSC aumenta en segundos en 2-4\% alcanzando un equilibrio en aproximadamente 2 minutos (figura 5). La respuesta cerebrovascular al cambio de la $\mathrm{PaCO}_{2}$ se atenúa con PAM bajas y aumenta con la hipoxia moderada, volviendo a disminuir frente a hipoxia severa, permaneciendo anormalmente baja después de la injuria hipóxica. En niños, la vasorreactividad al $\mathrm{CO}_{2}$ sería mayor respecto a adultos con el teórico mayor riesgo de hiperemia y edema frente a la hipercapnia ${ }^{18,20-23}$.

En el período post TEC se produce un deterioro transitorio de la vasorreactividad al $\mathrm{CO}_{2}$ la que se recupera entre los 4 a 7 días post injuria, situación que podría provocar isquemia, hiperemia o HTEC dependiendo del nivel de $\mathrm{PaCO}_{2}$. Adicionalmente, se ha determinado que con FSC basalmente más bajos, la vasorreactividad al $\mathrm{CO}_{2}$ también sería menor y en niños cambios en el $\mathrm{FSC}<2 \%$ respecto a los cambios de la $\mathrm{CO}_{2}$ se correlacionaría con un mal resultado neurológico.

En este contexto se explicaría el por qué la mantención de una $\mathrm{PaCO}_{2}$ dentro de rangos normales es tan importante en el TEC y por qué la hiperventilación e hipocapnia severa podría ser riesgosa y no efectiva en su tratamiento $^{18,22,24-27}$.

Por otro lado, la $\mathrm{PaO}_{2}$ tendría una influencia más lenta y menor sobre el FSC respecto de la $\mathrm{PaCO}_{2}$. Una $\mathrm{PaO}_{2}>50 \mathrm{mmHg}$ provocaría un mínimo cambio en el FSC, sin embargo, bajo este valor se produciría vasodilatación cerebral con importante aumento del FSC para mantener una adecuada entrega de $\mathrm{O}_{2}$, alcanzando un equilibrio recién a los 6 min de establecida la hipoxemia (figura 5) $)^{18,20,22}$.
El acoplamiento entre el Flujo sanguíneo cerebral y el metabolismo cerebral es quizás el mecanismo de control más importante sobre la circulación cerebral, si bien se desconoce el mecanismo exacto de esta interacción. Así, un bajo FSC determinará una limitación en la tasa metabólica y un mayor metabolismo provocará un aumento en el FSC. Esto último estaría determinado por un aumento en la neurotransmisión excitatoria, un mayor número de sinapsis, un mayor nivel de síntesis proteica y cambios en los sustratos metabólicos, todo lo cual sería independiente de los niveles locales tisulares de oxígeno. El acoplamiento FSC-metabolismo no está bien estudiado aun en niños ${ }^{18,22}$.

Posterior al TEC el $\mathrm{CMRO}_{2}$ disminuye en forma constante y esta disminución es proporcional al grado del coma. Si la regulación metabólica se mantiene intacta, existirá un buen acoplamiento entre el $\mathrm{CMRO}_{2}$ y el FSC con una $\mathrm{CEO}_{2}$ que no variará, pues existirá una disminución proporcional del FSC frente al menor $\mathrm{CMRO}_{2}$, es decir, habrá un adecuado balance entre la entrega y el consumo de $\mathrm{O}_{2}$. Esta situación ocurriría en aproximadamente el $45 \%$ de los pacientes con TEC ${ }^{12,28}$. Sin embargo, en otro porcentaje de pacientes se desarrollará un desacoplamiento entre el FSC y el metabolismo cerebral resultando en isquemia (FSC menor a demanda cerebral de $\mathrm{O}_{2}$ ) o hiperemia cerebral (FSC en exceso respecto al $\mathrm{CMRO}_{2}$ ). Se ha observado que en niños con TEC la velocidad de flujo en la arteria cerebral media es menor respecto a niños sin TEC, lo que supondría que la hipoperfusión (FSC $<25 \mathrm{ml} / 100 \mathrm{~g} / \mathrm{min}$ ) representaría la alteración dominante, con el consiguiente riesgo de isquemia cerebral y malos resultados; si bien el
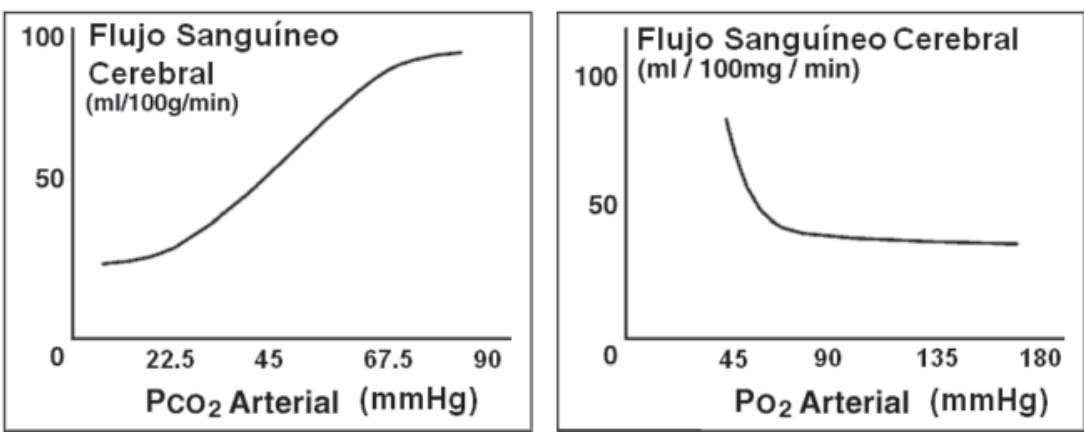

Figura 5. Efecto de la $\mathrm{PaCO}_{2}$ y $\mathrm{PaO}_{2}$ sobre el flujo sanguíneo cerebral. 
FSC también puede ser normal o alto con el riesgo de hiperemia o hemorragia ${ }^{18,22}$. Así, en el período precoz post TEC la constante disminución del $\mathrm{CMRO}_{2}$ puede acompañarse de un FSC variable dependiendo del buen acoplamiento metabolismo-flujo cerebral. En el TEC se describe un patrón estereotipado de alteraciones hemodinámicas, las que transcurrirían en 4 fases (figura 6) 18,12,22,28-30:

Fase 1: Hipoxia cerebral oligohémica o Perfusión límite, ocurre en general, durante las primeras 6 a 12 horas post TEC y puede llegar a niveles isquémicos en alrededor del 13\% de los pacientes. La Tomografía Axial Computarizada (TAC) mostrará un cerebro hipodenso. En esta fase se produce un desacoplamiento entre el $\mathrm{CMRO}_{2}$ y el FSC siendo este último menor a las necesidades metabólicas del cerebro, es decir, la $\mathrm{CDO}_{2}$ estará disminuida. El origen de esto pareciera ser el aumento de la resistencia vascular distal (microcirculación) de causa multifactorial: compresión micro- vascular extrínseca por podocitos astrocitarios edematosos y dañados, aumento de sustancias vasoactivas tales como calcio, catecolaminas, prostaglandinas, neuropéptido $\mathrm{Y}$; aumento de hemoglobina (vasoconstrictora) por hemorragia subaranoidea y trombosis intravascular. En condiciones de baja entrega de oxígeno (anemia, hipoxemia, hipotensión, etc.) el cerebro responderá compensatoriamente aumentando la $\mathrm{CEO}_{2}$ con caída de la Sat $\mathrm{BYO}_{2}$. Si el FSC se reduce severamente y por un tiempo suficiente, la $\mathrm{CEO}_{2}$ ya no podrá caer más $(>40 \%)$ y se producirá isquemia irreversible e infarto cerebral. Si en este punto se aumenta la $\mathrm{DO}_{2}$ la extracción de $\mathrm{O}_{2}$ no aumentará por existir un daño tisular permanente. Esto se reflejará en un aumento en la $\mathrm{Sat}_{\mathrm{BYO}}$ y en una caída de la $\mathrm{CEO}_{2}$ (células infartadas no extraerán $\mathrm{O}_{2}$ ). Para prevenir la isquemia la terapia deberá dirigirse a aumentar la $\mathrm{CDO}_{2}$ (corregir anemia, hipotensión, hipoxemia, hipocapnia, etc.) y/o a disminuir la $\mathrm{CMRO}_{2}$ (controlar agitación, convulsiones, temperatura, etc.).

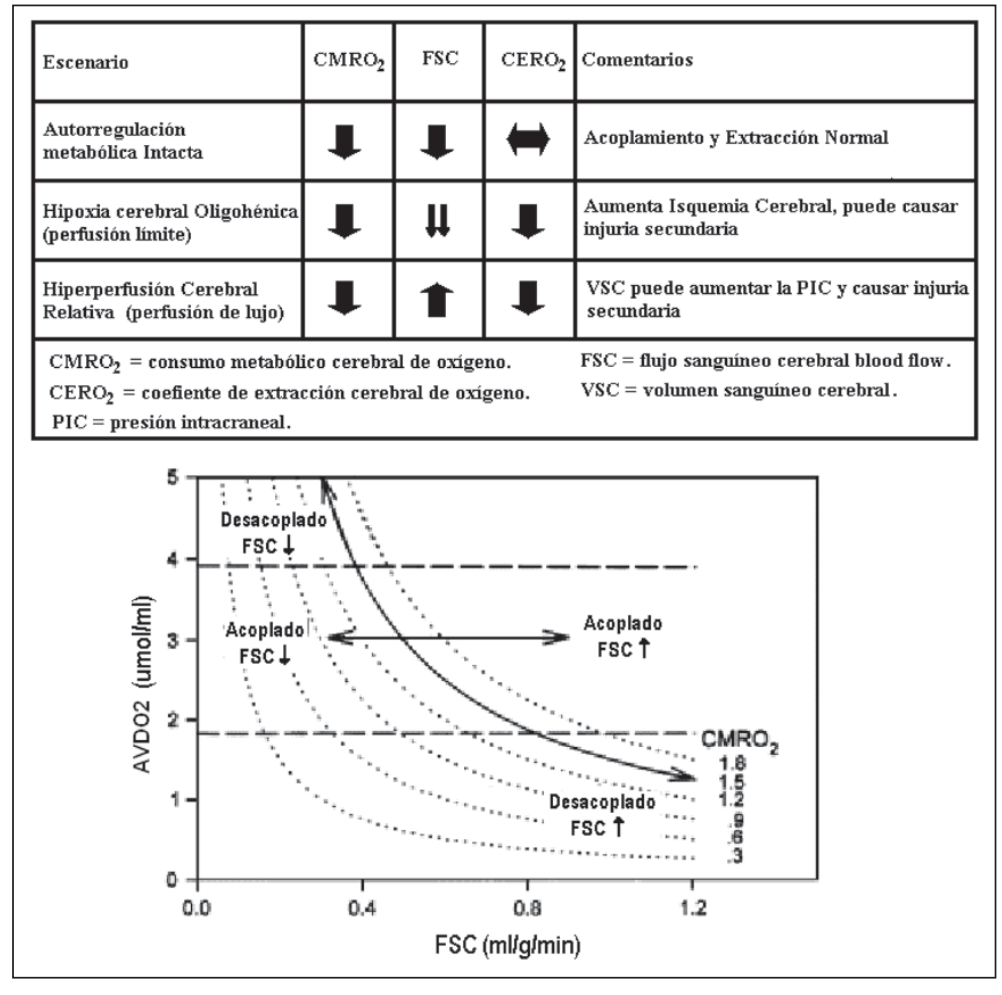

Figura 6. Escenarios post TEC. Adaptado de: Slavik R, Rhoney D: Pharmacologycal management of severe Traumatic Injury: An evidence-Based Review. J Inform Pharm 2000; 3: 309-35; Chesnut, R. Care of Central Nervous System Injuries. Surg Clin North Am 2007; 87: 119-56. 
Fase 2: Perfusión de lujo o hiperperfusión cerebral relativa, es menos frecuente de lo que se pensaba en la población pediátrica (incidencia actualmente estimada en 20-25\%), y aparecería entre las 24 a 96 horas de producido el TEC, alcanzando su peak a las 48-72 horas, aunque en algunos pacientes puede observarse posterior a este período. Aquí el FSC aumentará por hiperemia cerebral reactiva (a pesar de la disminución del $\mathrm{CMRO}_{2}$ ) favoreciendo la producción de edema cerebral y el aumento de la PIC (aumenta el VSC), condición que se observaría en el 15\% de los pacientes con TEC grave y que se correlacionaría con un mal resultado. Entre las causas de esto se contarían la generación de metabolitos vasodilatadores (ácido láctico, neuropéptidos y adenosina) post daño cerebral, además de la disfunción del músculo liso vascular inducido mecánicamente (vasoparálisis) que determinará la disminución de la RVC y el aumento del FSC. La TAC mostrará un cerebro hiperdenso, la Sat $\mathrm{BYO}_{2}$ será elevada $\left(>70 \%\right.$ ) y la $\mathrm{CEO}_{2}$ baja. La terapia deberá dirigirse a disminuir el volumen sanguíneo cerebral.

Fase 3: Vasoespasmo, ocurriría posterior a la fase hiperémica y hasta el día 14 de la injuria. Se produce una mala perfusión por caída del FSC y se correlacionaría con un mal outcome si alcanza niveles isquémicos. Tendría una baja incidencia en la población pediátrica, describiéndose entre el 0 al 19\% de los pacientes pediátricos con TEC.

Fase 4 o de recuperación: Comenzaría a las 2 a 3 semanas del trauma durando semanas a meses.

La importancia de reconocer estas fases radica en las implicancias terapéuticas en cada una de ellas, si bien otros factores influirán en la evolutividad del edema y la PIC.

La viscosidad de la sangre también tiene influencia sobre el FSC. La viscosidad es función del hematocrito y la hemodilución mejoraría el FSC por una mejor reología de la sangre en los vasos y también compensatoriamente por la disminución de la $\mathrm{CDO}_{2}$. Sin embargo, en el TEC se desconoce el valor del hematocrito ideal $^{18}$.

\section{Autorregulación cerebral}

La autorregulación cerebral ${ }^{18,20-23}$ es un mecanismo homeostático que consiste en la modificación de la resistencia vascular arteriolar (vasoconstricción/vasodilatación) en forma proporcional al valor de presión arterial media (PAM), dentro de un rango de presión, con el fin de mantener un flujo sanguíneo constante y acorde a las necesidades cerebrales de $\mathrm{O}_{2}$, y de proteger contra la isquemia y la hiperemia cerebral. En adultos sanos el rango o zona de autorregulación para la PAM es entre los 60 y $160 \mathrm{~mm} \mathrm{Hg}$ y para la Presión de perfusión cerebral (PPC) es entre 50 y $150 \mathrm{mmHg}$. Por sobre o bajo estos valores la resistencia vascular cerebral se torna dependiente de los valores de presión (figura 7), por lo que con hipotensión existirá riesgo de isquemia cerebral y con hipertensión riesgo de hiperemia y edema cerebral.

Destacable es que la capacidad autorregulatoria es mayor para la vasodilatación que para la vasoconstricción -hasta $65 \%$ del diámetro basal y hasta 8 a $10 \%$ del diámetro basal, respectivamente-, lo que significa que existe un mayor aumento del FSC frente a situaciones que determinen riesgo de isquemia (Ej.: hipotensión más que hipertensión). En niños entre los $6 \mathrm{~m}$ y 1 año el límite inferior de autorregulación se ha cifrado en $60 \pm 9 \mathrm{mmHg}$, por lo que debería evitarse cifras de PAM menores a este rango para impedir el desarrollo de isquemia cerebral.

Dentro de los mecanismos que actuarían en esta autorregulación se cuentan:

- Mecanismos miogénicos, mediante la capacidad intrínseca del músculo liso vascular para cambiar el diámetro en respuesta a cambios en la presión transmural.

- Mecanismos neurogénicos, a través de nervios de los vasos de mediano calibre que por activación simpática $\alpha$-adrenérgica provocarían la vasoconstricción.

- Mecanismos metabólicos en que los vasos pequeños alteran la respuesta vasomotora según condiciones del microambiente local (ejemplo, concentración de $\mathrm{O}_{2}, \mathrm{CO}_{2}, \mathrm{pH}$, etc.). 
La incidencia de alteración de la autorregulación en el TEC depende de su severidad. En adultos se observa en el $28 \%$ de los pacientes con TEC moderado y en el $67 \%$ de los con TEC severo y en los niños en el $17 \%$ de los TEC leve y en el $42 \%$ de los severos, viéndo-

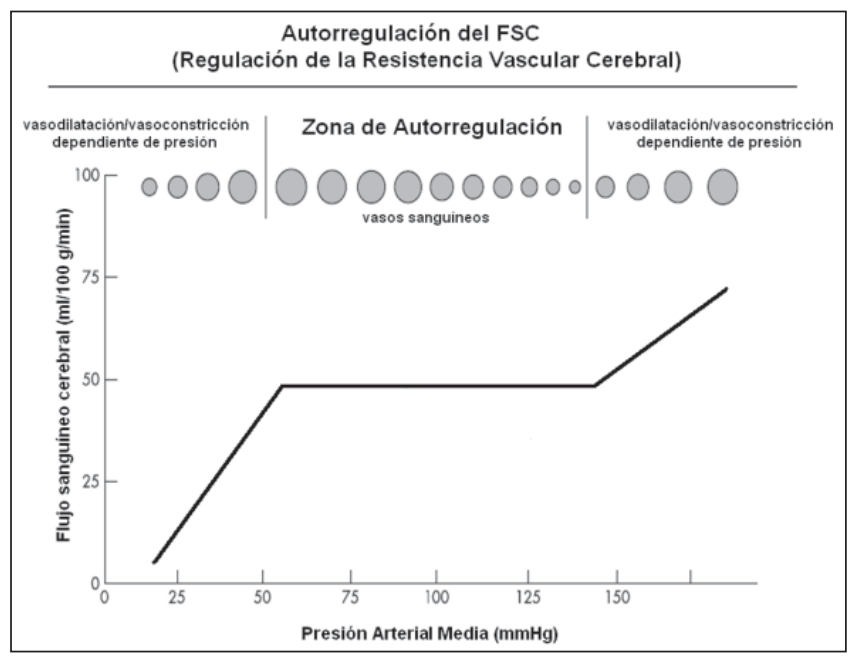

Figura 7. Autorregulación cerebral y FSC.
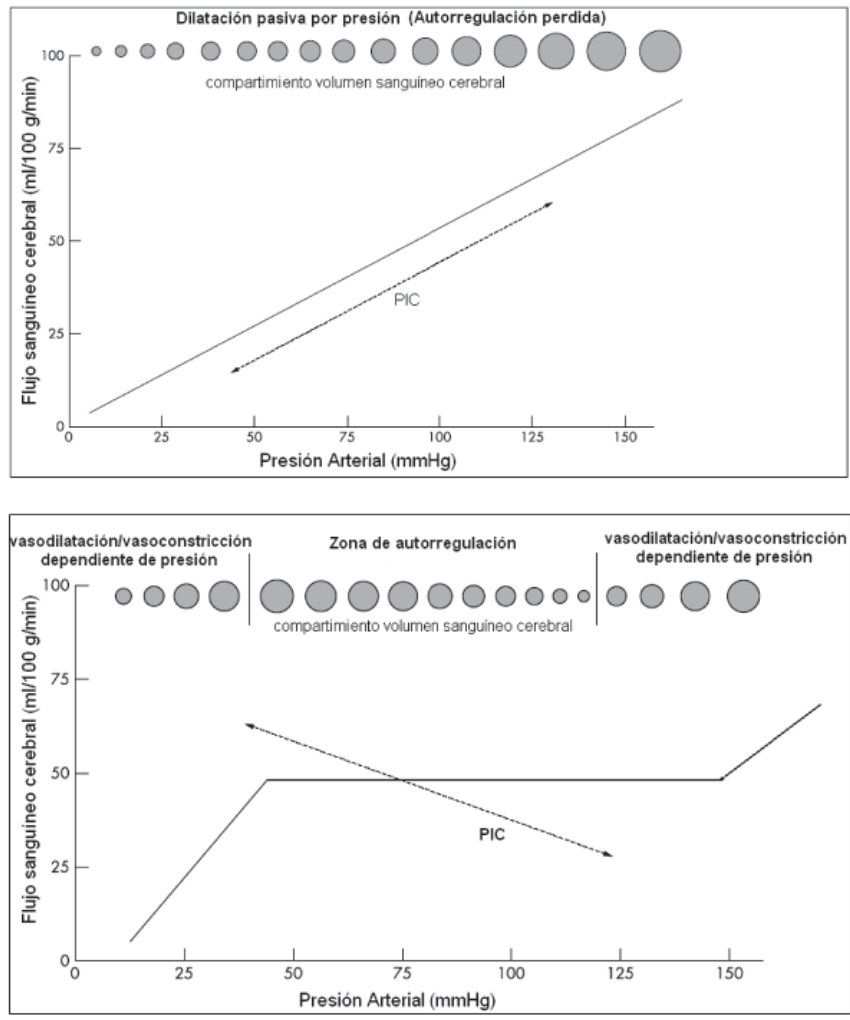

Figura 8. Autorregulación y relación con la PIC. se severamente afectada en niños con TEC por maltrato infantil. Si la autorregulación se ve alterada cifras bajas y altas de presión se asociarán a riesgo de isquemia e hiperemia respectivamente con ambas contribuyendo al desarrollo de edema cerebral. Por otro lado, la presencia de hiperemia se asociaría a alteración de la autorregulación y a malos resultados neurológi$\cos ^{18,21-23,31-33}$.

La autorregulación representa un espectro continuo de respuestas adaptativas de la resistencia cerebrovascular a los cambios en la PPC, no es una condición estática y puede deteriorarse en pacientes con autorregulación inicialmente intacta. Se piensa que este deterioro se correlacionaría con la pérdida de óxido nítrico o la disminución de sensibilidad a este. La alteración en la autorregulación puede ser mínima o incluso estar completamente perdida, puede ser unilateral o bilateral, puede ser diferente entre ambos hemisferios (se describen diferencias hemisféricas en hasta un $40 \%$ en las lesiones focales) y puede existir alteración de la autorregulación en los hemisferios cerebrales sin evidencia de lesión a la imagenología. Estudios han demostrado que la autorregulación es variable, pudiendo deteriorarse incluso hasta el día 9 de la injuria lo que se asociaría a empeoramiento del TEC. Sin embargo, la recuperación de la autorregulación puede demorarse hasta la segunda semana post TEC severo lo que ocurriría principalmente en pacientes con un bajo puntaje en la GCS inicial, en quienes presentan una injuria cerebral difusa, en quienes evolucionan con una PIC persistentemente elevada y quienes presentan un outcome neurológico desfavorable a los 6 meses de evolución. Esta situación sugeriría considerar en algunos pacientes la mantención de una terapia dirigida a una PPC adecuada por un período de -incluso- hasta 2 semanas ${ }^{18,21-23,31-33}$. 
Con la autorregulación alterada una reducción en la PAM puede determinar una disminución en el FSC y en la PPC con el riesgo de isquemia. Una PAM baja determinará vasodilatación, aumento del volumen de sangre cerebral con el consiguiente aumento de la PIC. El aumento de la PIC determinará una mayor disminución de la PPC desencadenando más vasodilatación cerebral, en un círculo vicioso que se ha denominado cascada vasodilatadora. Lo opuesto ocurre con una PAM elevada en lo que se denomina cascada vasoconstrictora. En ambas situaciones será necesario reconocer la noxa e implementar la intervención específica para detener el ciclo y evitar edema/ isquemia cerebral respectivamente (figuras $8 \mathrm{y}$ 9) ${ }^{18,20,22,34}$.

La autorregulación deteriorada se ha asociado a un mal resultado en el TEC pediátrico, pero aun existe poca información en niños ${ }^{22}$.

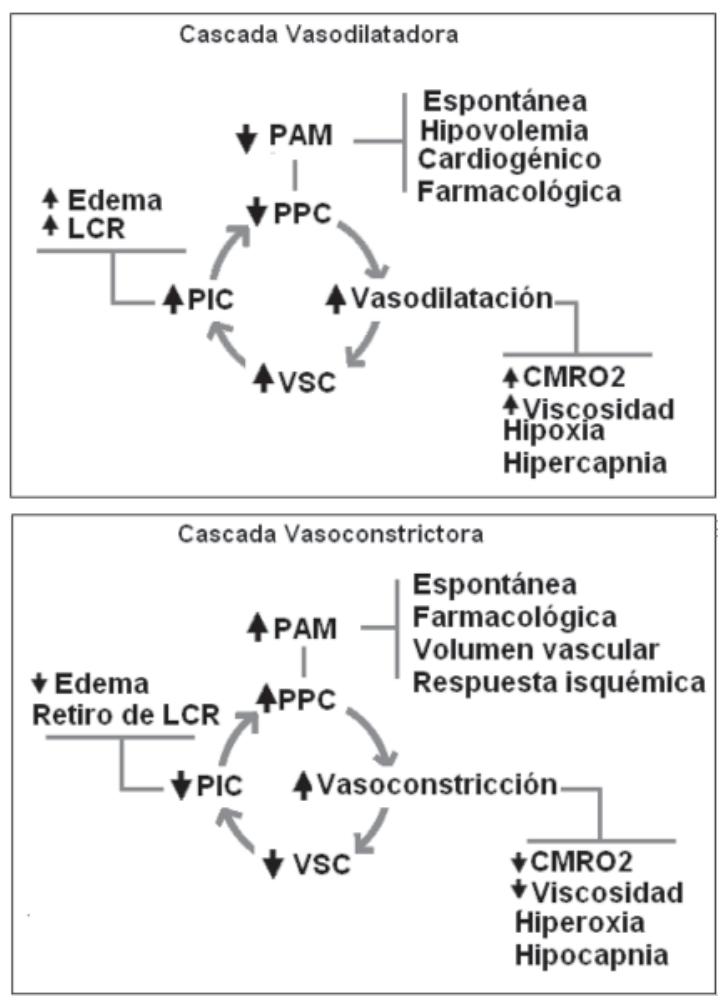

Figura 9. Cascada vasodilatadora y vasoconstrictora. Adaptado de: Walters F: Intracranial Pressure and Cerebral Blood Flow. In www.nda.ox.ac.uk/wfsa/html/u08/u08_013.htm.

\section{Edema cerebral}

A diferencia de los adultos, los niños tienen una mayor susceptibilidad a desarrollar edema cerebral frente a diversas injurias y noxas por poseer basalmente un mayor FSC, $\mathrm{CMRO}_{2}$, consumo metabólico de glucosa y vasorreactividad al $\mathrm{CO}_{2}$, además de una menor RVC basal. Estas particularidades fisiológicas son las determinantes de que el enfrentamiento terapéutico en el TEC deba ser aun más proactivo y agresivo en la población pediátrica respecto a la de adultos ${ }^{18,21-23}$.

Post TEC puede desarrollarse edema cerebral el cual provoca HTEC y predispone a isquemia y herniación cerebral si no es controlada a tiempo. El edema cerebral es producto del desarrollo de edema propiamente tal, de un VSC aumentado por hiperemia y congestión vascular o de una combinación de ambos. El edema propiamente tal se genera por 3 mecanismos $^{10,11}$ :

- Edema celular o citotóxico, afecta principalmente a los podocitos astrocitarios, aunque también a las neuronas y a las células endoteliales. Ocurre por falla en los mecanismos homeostáticos de la mantención del tamaño celular pues fallan mecanismos energéticos (ATP).

- Injuria de la BHE y edema vasogénico, la BHE es más vulnerable a la ruptura en cerebros inmaduros. La mayor alteración de la BHE se produciría dentro de las primeras horas posteriores al daño, e injurias tales como la hipoxemia e hipotensión la empeorarían. Además del daño mecánico, también mediadores bioquímicos tales como metabolitos de la ciclooxigenasa, radicales libres y citoquinas provocarían daño en la BHE.

- Edema osmolar, contribuiría en forma importante al desarrollo del edema en áreas de contusión y necrosis. Se produciría por la degradación de macromoléculas presentes en las áreas contundidas o necróticas lo que aumentaría la carga osmolar en la zona. Al mejorar la BHE se provocaría una gradiente de presión que determina la acumulación de agua y con ello el desarrollo de un importante edema. 
El edema cerebral postraumático, cuando aparece, ocurre en tiempos variables observándose frecuentemente el peak dentro de las primeras 72 horas post injuria (por el patrón hemodinámico del FSC ya descrito). La aparición de edema cerebral tardío e $\operatorname{HTEC~}\left(5^{\circ}-7^{\circ}\right.$ día post trauma) tendría una frecuencia aparentemente baja, aunque la evolutividad del edema y de la PIC post TEC no es bien conocida. Pese a lo anterior, el estado del VSC y del edema puede ser inferido de la imagen cerebral al TAC $\mathrm{TA}^{35-37}$.

En una serie de 201 pacientes con TEC grave se observó que la HTEC variaba ampliamente, si bien la mayoría de los pacientes presentaban PIC elevadas dentro de los primeros 5 días. Sin embargo, aproximadamente 1/5 de los casos evolucionaba con PIC elevadas posterior a este período por mecanismos no del todo claros. A partir de esto, los autores sugerían monitorizar la PIC por un mínimo de 5 días post injuria ${ }^{35}$. Por otro lado, O'Phelan et al en una revisión retrospectiva de 77 pacientes observaron 4 patrones evolutivos de la PIC: precoz (dentro de las 72 horas del trauma), tardío (posterior a las 72 horas), bimodal (aumento PIC precoz, normalización y aumento posterior a las 72 horas) y continuo (PIC siempre elevada). El 17\% de este grupo de pacientes tuvo un aumento tardío de la PIC con el peak de edema al $7^{\circ}$ día post injuria. No se logró identificar un factor predictor estadísticamente significativo de aumento de PIC tardío, por lo que también estos autores sugerían mantener la monitorización de la PIC por un período prolongado considerando el riesgo de aparición de edema cerebral tardío ${ }^{36}$.

\section{Conclusiones}

Si bien la prevención primaria es la estrategia para disminuir rotundamente la morbimortalidad asociada al TEC, el principal objetivo de su manejo es evitar el desarrollo de la injuria secundaria, con el fin de impedir o minimizar el daño neurológico secuelar. Por lo mismo, es fundamental reconocer las diferencias fisiológicas en la población pediátrica respecto a la de adultos de manera tal de enten- der mejor la fisiopatología subyacente al TEC y así instaurar un tratamiento racional precoz, dirigido a prevenir, reconocer y tratar las noxas asociadas con un mal resultado neurológico.

\section{Referencias}

1.- Cáceres A, Wegner A, Montecinos T, Bongain J. TEC en Pacientes Pediátricos. En: Guía clínica Atención de Urgencia del Traumatismo craneoencefálico. 2007. Serie Guías Clínicas MINSAL No 49, págs. 23-38.

2.- Teasdale G, Jennett B: Assessment of coma and impaired consciousness. A practical scale. Lancet 1974; 2: 81-4.

3.- Reilly PL, Simpson DA, Sprod R, et al: Assessing the conscious level in infants and youg children: a pediatric version of the Glasgow Coma Scale. Childs Nerv Syst 1988; 4: 30-3.

4.- ASOCIMED: Pautas Diagnóstico-Terapéuticas para la práctica Clínica. Tema, condición o Patología: Traumatismo Encefalocraneano. En http://www.asocimed. cl/Guias\%20Clinicas/neurocirugia/traumatismoencefalocraneano.html. Acceso el 30 de Septiembre de 2010.

5.- Nalegach, ME. Magnitud y Epidemiología de los Traumas y Accidentes en Chile. En: http://www.medwave. cl/atencion/infantil/FUDOCV2003/2/1.act. Acceso el 04 de Noviembre de 2008.

6.- Días MS: Traumatic brain and spinal cord injury. 2004. Pediatr Clin N Am 51: 271-303.

7.- Tasker RC: Head and Spinal Cord Trauma. En Rogers' Textbook of Pediatric Intensive Care. David G. Nichols (Ed.). Lippincott Williams \& Wilkins. 4 Edición, Capítulo 56, págs. 888-911.

8.- Chesnut RM: Management of brain and spine injuries. 2004. Crit Care Clin 20: 25-55.

9.- Kochanek P, Clark R, Ruppel R, et al: Biochemical, cellular and molecular mechanisms in the evolution of secondary damage after severe traumatic brain injury in infants and children: Lessons learned from the bedside. Pediatr Crit Care Med 2000; 1: 4-9.

10.- Bayir H, Kochaneck P, Clark R: Traumatic Brain injury in infants and children. Mechanism of secondary damage and treatment in the intensive care unit. Crit Care Clin 2003; 19: 529-49.

11.- Park E, Bell JD, Baker AJ: Traumatic brain injury: Can the consequences be stopped? CMAJ 2008; 178 (9): 1163-70.

12.- Slavik R, Rhoney D: Pharmacologycal management of severe Traumatic Injury: An evidence-Based Review. J Inform Pharm 2000; 3: 309-35 
13.- Adelson PD, Bratton SL, Carney NA, et al; American Association for Surgery of Trauma; Child Neurology Society; International Society for Pediatric Neurosurgery; International Trauma Anesthesia and Critical Care Society; Society of Critical Care Medicine; World Federation of Pediatric Intensive and Critical Care Societies: Guidelines for the acute medical management of severe traumatic brain injury in infants, children, and adolescents. Chapter 8: Cerebral Perfusion Pressure. Pediatr Crit Care Med 2003; 4 (3 Suppl): S31-3.

14.- Bratton S, Chesnut R, Ghajar J, et al: IX. Cerebral Perfusion thresholds. J Neurotraum 2007; 24 (Suppl 1): S59-S64.

15.- Steiner L, Andrews $P$ : Monitoring the injured brain: IPC and CFB. Br J Anaesth 2006; 97: 26-38.

16.- Adelson PD, Bratton SL, Carney NA, et al; American Association for Surgery of Trauma; Child Neurology Society; International Society for Pediatric Neurosurgery; International Trauma Anesthesia and Critical Care Society; Society of Critical Care Medicine; World Federation of Pediatric Intensive and Critical Care Societies: Guidelines for the acute medical management of severe traumatic brain injury in infants, children, and adolescents. Chapter 6 . Threshold for treatment of intracranial hypertension. Pediatr Crit Care Med 2003; 4 (3 Suppl): S25-7.

17.- Wegner AA, Wilhelm BJ, Darras E: Traumatismo encefalocraneano: Conceptos fisiológicos y fisiopatológicos para un manejo racional. Rev Chil Ped 2003; 74 (1): 16-30.

18.- Udomphorn Y, Armstead W, Vavilala M: Cerebral Blood Flow and Autoregulation After Pediatric Traumatic Brain Injury. Pediatr Neurol 2008; 38: 225-34.

19.- Greisen G: Cerebral blood flow and energy metabolism in the developing brain. En: Fetal and Neonatal Neurology and Neurosurgery . Philapdelphia: Editorial Elsevier 2008; 171-91.

20.- Walters F: Intracranial Pressure and Cerebral Blood Flow. In www.nda.ox.ac.uk/wfsa/html/u08/u08_013. htm. Acceso Diciembre 2010.

21.- Rangel-Castilla L, Gasco J, Nauta HJ, Okonkwo DO, Robertson CS: Cerebral pressure autoregulation in traumatic brain injury. Neurosurg Focus 2008; 25 (4): E7.

22.- Philip S, Udomphorn Y, Kirkham FJ, Vavilala M: Cerebrovascular Pathophysiology in Pediatric Traumatic Brain Injury. J Trauma 2009; 67 (2): S128-34.

23.- Vavilala MS, Lee LA, Boddu K, et al: Cerebral autoregulation in pediatric traumatic brain injury. Pediatr Crit Care Med 2004; 5: 257-63.
24.- Lee JH, Kelly DF, Oertel M, et al: Carbon dioxide reactivity, pressure autoregulation, and metabolic suppression reactivity after head injury: a Transcranial Doppler study. J Neurosurg 2001; 95: 222-32.

25.- Adelson PD, Clyde B, Kochanek PM, Wisniewski SR, Marion DW: Cerebrovascular Response in Infants and Young Children following Severe Traumatic Brain Injury: A Preliminary Report. Pediatr Neurosurg 1997; 26: 200-7.

26.- Stocchetti N, Maas A, Chieregato A, Van der Plas A: Hyperventilation in Head Injury A Review. Chest 2005; 127: 1813-27.

27.- Adelson PD, Bratton SL, Carney NA, et al; American Association for Surgery of Trauma; Child Neurology Society; International Society for Pediatric Neurosurgery; International Trauma Anesthesia and Critical Care Society; Society of Critical Care Medicine; World Federation of Pediatric Intensive and Critical Care Societies: Guidelines for the acute medical management of severe traumatic brain injury in infants, children, and adolescents. Chapter 12. Use of hyperventilation in the acute management of severe pediatric traumatic brain injury. PCCM 2003; 4 (3): S45-8.

28.- Mandera M, Larysz D, Wojtacha M: Changes in cerebral hemodynamics assessed by transcranial Doppler ultrasonography in children after head injury. Childs Nerv Syst 2002; 18: 124-8.

29.- White J, Farukhi Z, Bull C, et al: Predictors of outcome in severely head injured children. Crit Care Med 2001; 29: 534-40.

30.- Martin N, Patwardhan R, Alexander M, et al: Characterization of cerebral hemodynamic phases following severe head trauma: hypoperfusion, hyperemia, and vasospasm. J Neurosurg 1997; 87: 9-19.

31.- Vavilala MS, Muangman S, Waitayawinyu P, et al: Neurointensive care: impaired cerebral autoregulation in infants and young children early after inflicted traumatic injury: a preliminary report. J Neurotrauma 2007; 24 : 87-96.

32.- Tontisirin N, Armstead W, Waitayawinyu P, et al: Change in cerebral autoregulation as a function of time in children after severe traumatic brain injury: a case series. Childs Nerv Syst 2007; 23: 1163-9.

33.- Sviri G, Aaslid R, Douville C, Moore A, Newell D: Time course for autoregulation recovery following severe traumatic brain injury J. Neurosurg 2009; 24: 1-6.

34.- Rose J, Mayer S: Optimizing Blood Pressure in Neurological Emergencies. Neurocrit Care 2004; 1: 287-99.

35.- Stochetti N, Colombo A, Ortolano F, et al: Time Course of Intracranial Hypertension after Traumatic Brain In- 
WEGNER A. y cols.

jury. J Neurosurg 2007; 24: 1339-46.

36.- O'Phelan K, Park D, Efird J, et al: Patterns of increased intracranial pressure after severe Traumatic Brain Injury. Neurocrit Care 2009; 10: 280-6.
37.- Reithmeier T, Speder B, Pakos P, et al: Delayed bilateral craniectomy for treatment of traumatic brain swelling in children: case report and review of the literature. Childs Nerv Syst 2005; 21: 249-53. 